\title{
Delay of neuropathic pain sensitization after application of dexamethasone-loaded implant in sciatic nerve-injured rats
}

\author{
Leandro Francisco Silva Bastos', Juliana Priscila Vago ${ }^{2}$, Thaís Rolla Caux ${ }^{2}$, \\ Bruna Lopes Costa ${ }^{2}$, Adriana Martins Godin ${ }^{2}$, Raquel Rezende Menezes ${ }^{2}$, Roberta Ribas Pena ${ }^{1}$, \\ Armando Silva-Cunha ${ }^{\circledR 2}$, Lirlândia Pires Sousa ${ }^{2}$, Renes Resende Machado ${ }^{\circledR 2}$, \\ Márcio Flávio Dutra Moraes ${ }^{1}$, Sílvia Ligório Fialho ${ }^{\circledR *}$, Márcio Matos Coelho²
}

\author{
${ }^{1}$ Biological Sciences Institute, Federal University of Minas Gerais (UFMG), Belo Horizonte, MG, Brazil, ${ }^{2}$ Faculty of \\ Pharmacy, Federal University of Minas Gerais (UFMG), Belo Horizonte, MG, Brazil, ${ }^{3}$ Pharmaceutical Research and \\ Development, Ezequiel Dias Foundation, Belo Horizonte, MG, Brazil
}

\begin{abstract}
Neuroimmune interactions underlying the development of pain sensitization in models of neuropathic pain have been widely studied. In this study, we evaluated the development of allodynia and its reduction associated with peripheral antineuroinflammatory effects induced by a dexamethasone-loaded biodegradable implant. Chronic constriction injury (CCI) of the sciatic nerve was performed in Wistar rats. The electronic von Frey test was applied to assess mechanical allodynia. The dexamethasone-loaded implant was placed perineurally at the moment of CCI or 12 days after surgery. Dorsal root ganglia (DRG; L4-L5) were harvested and nuclear extracts were assayed by Western blot for detection of nuclear factor (NF)-кB p65/RelA translocation. Dexamethasone delivered from the implant delayed the development of allodynia for approximately three weeks in CCI rats when the implantation was performed at day 0 , but allodynia was not reversed when the implantation was performed at day 12 . NF- $\kappa \mathrm{B}$ was activated in CCI rat DRG compared with naïve or sham animals (day 15), and dexamethasone implant inhibited p65/ RelA translocation in CCI rats compared with control. This study demonstrated that the dexamethasoneloaded implant suppresses allodynia development and peripheral neuroinflammation. This device can reduce the potential side effects associated with oral anti-inflammatory drugs.
\end{abstract}

Keywords: Chronic Constriction Injury. Dexamethasone/ pharmacology. Delivery device. Neuropathic Pain/ chemically induced. Nuclear Factor kappa B/ drug effects. Neuralgia/ chemically induced. NFkappa B/ drug effects. Sciatic Nerve/ injuries. Absorbable Implants/trends.

\section{INTRODUCTION}

Dexamethasone, a potent anti-inflammatory and immunosuppressant drug, inhibits fibrovascular tissue formation around sutures, infiltration of inflammatory cells, and thermal hyperalgesia when administered intraperitoneally before and after chronic constriction injury (CCI) in rats (Clatworthy et al., 1995). It is tempting to associate the antiallodynic effect induced by a steroidal anti-inflammatory drug (SAID) with its anti-inflammatory actions on the peripheral nervous

\footnotetext{
*Correspondence: S. L. Fialho. Pharmaceutical Research and Development, Ezequiel Dias Foundation. Rua Conde Pereira Carneiro, 80 - Gameleira - CEP 30510-010, Belo Horizonte / MG - Brazil. Tel: 55-31-3314 4986; Fax 55-31-3314 4766. E-mail address: silvia.fialho@funed.mg.gov.br
}

system. When rats with CCI have the sciatic nerves re-exposed and treated locally with a depot form of methylprednisolone at day 10 after injury, antiallodynic and antihyperalgesic effects are observed up to day 21 (Johansson, Bennett, 1997), thus providing evidence that SAIDs may act locally at the site of nerve injury to inhibit sensitization. On the other hand, spinally applied dexamethasone induces pro-nociceptive effects after peripheral nerve injury (Wang et al., 2004).

Immune activation and the subsequent release of immune mediators in the peripheral nervous system contribute to pain. A variety of peripheral immune cells, including mast cells, macrophages, and lymphocytes, are resident in nerves and/or recruited to sites of peripheral nerve injury. These cells produce a plethora of 
inflammatory mediators that sensitize or directly activate peripheral neurons to generate action potentials, leading to peripheral and central sensitization. The peripheral and central sensitization contributes to the development of allodynia and hyperalgesia, two hallmarks of neuropathic painful states (McMahon, Cafferty, Marchand, 2005; Perkins, Tracey, 2000). Proliferation of satellite cells occurs in dorsal root ganglia (DRGs), as well as infiltration of macrophages and T cells to this site (McMahon, Cafferty, Marchand, 2005). Different kinds of sciatic nerve injury lead to activation of nuclear factor $\kappa B(N F-\kappa B)$ in DRGs (Ma, Bisby, 1998). In non-stimulated cells, NF-kB consists of a heterodimer of subunits $\mathrm{p} 50$ and $\mathrm{p} 65$ that form a complex with $\mathrm{I} \kappa \mathrm{B}$, an inhibitory protein. Several endogenous or exogenous molecules can activate NF- $\kappa B$, mainly the heterodimers $\mathrm{p} 65 \mathrm{RelA} / \mathrm{p} 50$, which translocate to the nucleus, thereby modulating the expression of pro-inflammatory genes, such as those for inducible nitric oxide synthase, cyclo-oxygenase-2, adhesion molecules, cytokines, and chemokines (Kaltschmidt,

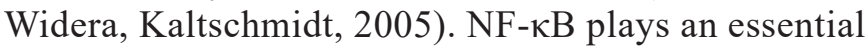
role in the gene expression of inflammatory mediators in neurons and glial cells, besides many other cell types. Transgenic inhibition of glial NF- $\kappa B$ inhibits hyperalgesia and allodynia in mice, and these effects are associated with reduced cytokine/chemokine production in DRGs (Fu et al., 2010).

To further study the peripheral antineuroinflammatory actions of SAIDs, in the present study we investigated the ability of a dexamethasone-loaded biodegradable implant to prevent the mechanical allodynia induced by sciatic injury in rats and its possible association with an effect on NF- $\kappa$ B activation in DRG. Biodegradable implants may be an effective dosage form since they may promote a controlled drug release at therapeutic levels for a prolonged period of time without frequent doses, avoiding undesirable targeting of healthy tissues and harmful side effects, as lower concentrations of drugs are administered when compared to oral formulations (Dash, Cudworth 2nd., 1998; Langer, 1998).

\section{MATERIAL AND METHODS}

Dexamethasone was purchased from Sigma-Aldrich (Brazil). Poly-lactide-co-glycolide copolymer (PLGA 50:50, PURASORB ${ }^{\circledR}$ PDLG 5004, inherent viscosity midpoint of $0.4 \mathrm{dl} / \mathrm{g}$ ) was a gift from Purac Biomaterials (Brazil). Acetonitrile HPLC grade was purchased from Merck Brasil (Brazil). Ultrapure water was obtained from Milli-Q, Millipore (USA). All other chemicals were of analytical grade.

\section{Preparation of dexamethasone-loaded biodegradable implants}

The implants were prepared according to the technique previously described by Fialho, Silva-Cunha (2005). Dexamethasone and the polymer PLGA 50:50 at a ratio 1:4 were dissolved in a mixture of distilled water and acetonitrile and the resulting solution was lyophilized $\left(-40^{\circ} \mathrm{C}\right.$, Pirani501 78/L, Edwards Ltda., Brazil). The powder obtained was molded into rods using a hot plate. The implants weighed on average 6.0 $\pm 0.1 \mathrm{mg}$, were $4.0 \pm 0.1 \mathrm{~mm}$ in length, and $1.0 \pm 0.05$ $\mathrm{mm}$ in diameter (Figure 1). The final concentration of dexamethasone dispersed in the polymeric matrix was $40 \% \mathrm{w} / \mathrm{w}$. Implants without drug were also prepared using the same technique.

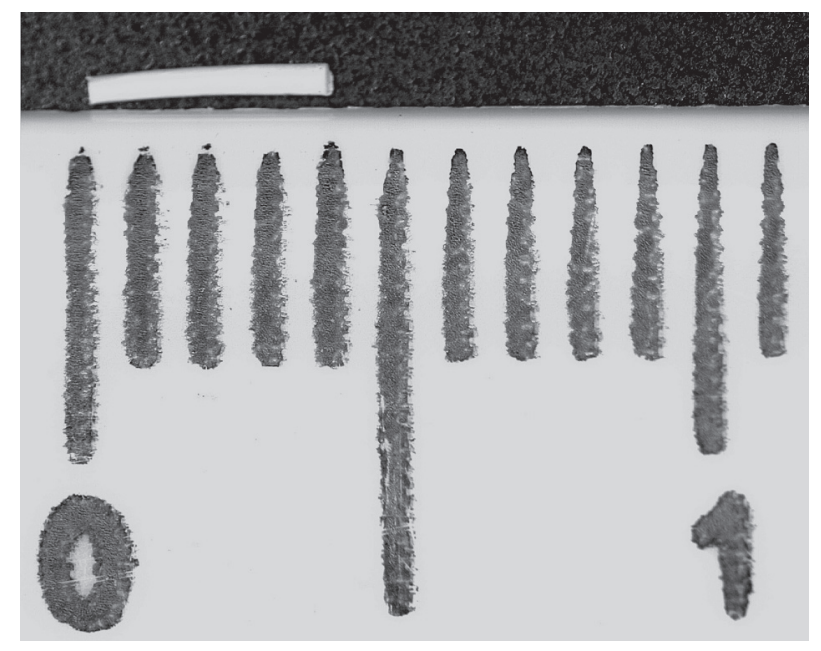

FIGURE 1 - Dexamethasone-loaded PLGA implant (weight: $6.0 \pm 0.1 \mathrm{mg}$; length: $4.0 \pm 0.1 \mathrm{~mm}$; diameter: $1.0 \pm 0.05 \mathrm{~mm})$.

\section{Animals}

All experiments were performed according to the ethical guidelines for the investigation of experimental pain in conscious animals and to the EU Directive 2010/63/EU for animal experiments. The study was approved by the Ethics Committee on Animal Experimentation of the Federal University of Minas Gerais (Protocol 168/2009).

Male Wistar rats (240-280 g) were used, with groups matched on body mass (approximately $260 \mathrm{~g}$ on average). The animals had free access to food and water and were maintained in a room with a $12 \mathrm{~h}$ light-dark cycle for $3 \mathrm{~d}$ before the experiment to allow for acclimatization. For three consecutive days before the experiment, the animals were habituated for approximately $30 \mathrm{~min}$ to the testing apparatuses in order to minimize stress-induced 
antinociception. The investigator performing behavioral tests was blinded to all treatments.

\section{Surgery and drugs}

Chronic constriction injury (CCI) of the right sciatic nerve was performed as previously described by Bennett, Xie (1988), under anesthesia induced by ketamine $(90 \mathrm{mg} / \mathrm{kg})$ and xylazine $(9 \mathrm{mg} / \mathrm{kg})$ hydrochlorides (both manufactured by Vetbrands; Paulínia, São Paulo, Brazil). Four ligatures with a flexible nylon thread were tied loosely around the sciatic nerve at mid-thigh level (Motta et al., 2011). Sham-operated animals underwent the same surgical procedure, save that the sciatic was not ligated. Dexamethasone-loaded PLGA implants or PLGAonly implants were placed perineurally at the moment of $\mathrm{CCI}$ or after re-exposure of the sciatic (under anesthesia) at day 12 after surgery.

\section{Electronic von Frey test for mechanical allodynia}

Rats were placed in acrylic cages with wire mesh floors $15 \mathrm{~min}$ before the start of testing. The test consisted of evoking a hind paw flexion reflex with a hand-held force transducer (electronic pressure-meter, Insight, Ribeirão Preto, Brazil) adapted to a $0.5-\mathrm{mm}^{2}$ polypropylene tip. The experimenter was trained to apply the tip perpendicularly to the central area of the hind paw with a gradual increase in pressure. The endpoint was characterized by the removal of the paw usually followed by flinching movements. After the paw withdrawal, the intensity of the pressure was automatically recorded. The mean withdrawal threshold was obtained by averaging 5 measurements. The animals were tested before and after surgeries and/ or treatments. The results are expressed by the absolute withdrawal threshold (in grams). These procedures were done similarly to those previously described elsewhere (Vivancos et al., 2004).

\section{NF-кB translocation evaluation}

$\mathrm{NF}-\kappa \mathrm{B}$ translocation was investigated by Western blotting analysis of nuclear cell extracts obtained from homogenized DRGs, as described elsewhere (Souza et al., 2009). Ipsilateral L4 and L5 DRGs were harvested and immediately frozen in liquid nitrogen. Briefly, the tissue was homogenized in ice-cold hypotonic lysis buffer (10 mM Tris pH 7.4, $10 \mathrm{mM} \mathrm{NaCl}, 3 \mathrm{mM} \mathrm{MgCl}, 0.002 \%$ $\mathrm{NaN}_{3}, 1 \mathrm{mM}$ PMSF, $0.1 \mathrm{mM}$ EGTA, $10 \mu \mathrm{M}$ aprotinin, $20 \mu \mathrm{M}$ leupeptine, $0.5 \mathrm{mM}$ DTT, $25 \mathrm{mM} \mathrm{NaF}$ ) and then homogenized for approximately $1 \mathrm{~min}$. Next, the same buffer was added $(500 \mu \mathrm{L})$ and the suspension was shaken in a vortex. The tissue was kept on ice for $15 \mathrm{~min}$. Next, Triton ${ }^{\circledR}$ X-100 (5\%) was added and the mixture was inverted gently three times and kept on ice for $5 \mathrm{~min}$. Centrifugation was performed $\left(6000 \mathrm{rpm}, 6 \mathrm{~min}, 4^{\circ} \mathrm{C}\right)$ and the supernatant containing the cytoplasmic protein extract was discarded. The remaining pellet was washed out with RSB 1X buffer and centrifuged $(9000 \mathrm{rpm}, 6 \mathrm{~min}$, $4{ }^{\circ} \mathrm{C}$ ). The supernatant was carefully removed to ensure the cytoplasmic extract separation and then the nuclear extract was re-suspended in $100 \mu \mathrm{L}$ buffer C $(20 \mathrm{mM}$ HEPES pH 7.4, $420 \mathrm{mM} \mathrm{NaCl}, 1.5 \mathrm{mM} \mathrm{MgCl} 2,0.01 \%$ $\mathrm{NaN}_{3}, 0.2 \mathrm{mM}$ EDTA, 25\% v/v glycerol, $1 \mathrm{mM}$ PMSF, $10 \mathrm{mM}$ aprotinin, $20 \mathrm{mM}$ leupeptin, $0.5 \mathrm{mM}$ DTT). This suspension was kept on ice for $45 \mathrm{~min}$, a period during which the liquid was shaken in a vortex for $15 \mathrm{~s}$ every 5 min. Afterwards, centrifugation was performed and the supernatant was stored at $-70^{\circ} \mathrm{C}$ for Western blot analysis. Nuclear extracts contents were determined by the Bradford method, using the Bio-Rad Protein Assay Kit (Bio-Rad Laboratories, Hercules, CA, USA). For immunoblotting, $15 \mu \mathrm{g}$ of protein from each sample was subjected to SDSpolyacrylamide gel electrophoresis on a $10 \%$ gel. Primary antibodies were anti-p65/Rel $A$ (Santa Cruz Biotechnology, Dallas, TX, USA; catalog \# sc-372; dilution 1:1000) and anti- $\beta$-actin (Sigma-Aldrich, Saint Louis, MO, USA; catalog \# A5316; dilution 1:5000), both diluted in PBS/ Tween and bovine serum albumin (5\%). Proteins were detected with horseradish peroxidase (HRP)-coupled goat antirabbit immunoglobulin G or HRP-coupled goat antimouse (sc-2357 and sc-2005, respectively; both diluted 1:3000 in PBS/Tween and 5\% skim milk), using chemiluminescence reagents (GE Healthcare, Piscataway, NJ, USA).

\section{Percentage of dexamethasone released from the implants}

The amount of dexamethasone released from the implants was calculated indirectly from the retrieved implants by high performance liquid chromatography.

The implants were withdrawn at day 15 after constriction injury, gently washed with distilled water, and then dissolved in a fixed volume of acetonitrile. The amount of dexamethasone was measured by high-performance liquid chromatography using the method described in the United States Pharmacopeia with a Waters apparatus equipped with an autosampler model 717 plus (Waters, Milford, MA, USA). A pump (model 515; Waters) was used at a constant low rate of $1.2 \mathrm{~mL} / \mathrm{min}$. A C-18 reverse-phase column $(3.9 \times 150 \mathrm{~mm})$ 
filled with octadecylsilane chemically bonded to porous silica ( $5 \mu \mathrm{m}$, Nova-Pak; Waters) was used. The mobile phase was a mixture of acetonitrile and ultra-filtrated water (45:55). An ultraviolet detector (model 2487; Waters) was used (wavelength: $254 \mathrm{~nm}$ ). The validation of the method showed the absence of interference of the polymer with the drug retention time, ruling out the risks of overestimation.

\section{Statistical analyses}

The number of animals used in each protocol was based on the mathematical relationship between the values of the studied effect, standard deviation, level of significance and the statistical test power. The behavioral and body mass results, expressed as mean \pm standard error of the mean, were analyzed by two-way repeated measures ANOVA followed by Bonferroni post hoc test, taking treatment and time as main effects (Figures 2 and 3 ). Image J 1.46r (NIH, USA) was used to semiquantitatively analyze the immunoblots, which were normalized to $\beta$-actin. ANOVA followed by Newman-Keuls post hoc test or $t$ test was used for comparisons of densitometric values (Figure 4). Values of $p<0.05$ were considered to show significant differences between means. The software Prism $^{\circledR} 5$ (GraphPad Software Inc., San Diego, CA, USA) was used for the analyses.

\section{RESULTS}

The perineural implant of dexamethasone delayed the development of allodynia when the implantation was performed simultaneously to CCI (i.e., at day 0; Figure 2A)

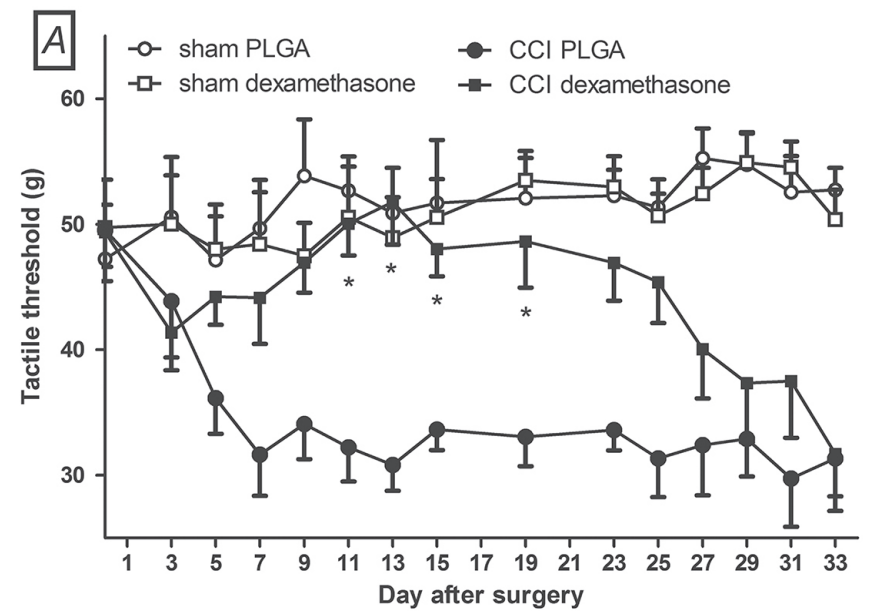

in rats, but not when the implantation was performed at day 12 (Figure 2B), when the allodynia was fully established. The percentage of dexamethasone release from the implants, assessed 15 days after implantation, was $66.4 \%$, i.e., approximately $1.6 \mathrm{mg}$.

Dexamethasone implantations, performed either at day 0 or day 12, prevented body mass gain (Figures $3 \mathrm{~A}$ and $3 \mathrm{~B}$, respectively), indicating that dexamethasone had been released and had induced its long-known catabolic effect (Bullock et al., 1972).

As expected, CCI upregulated p65/RelA translocation in DRG cells (Figures 4A and 4B). Taking into account the importance of NF- $\kappa B$ for the expression of several inflammatory mediators and the well-known effects induced by SAIDs on NF- $\kappa \mathrm{B}$ activation, we investigated the association between the prevention of allodynia development and peripheral antineuroinflammatory effect. Indeed, in rats subjected to dexamethasone implantation $(2.4 \mathrm{mg})$ at the moment of constriction, we observed reduced translocation of $\mathrm{NF}-\kappa \mathrm{B}$ in comparison with vehicle (PLGA)-treated rats (Figures 4C and 4D).

\section{DISCUSSION}

In the present study, we showed that a perineural implant containing dexamethasone, placed at the moment of CCI, delays the development of mechanical allodynia in rats. However, such an effect is not observed if the nerve is re-exposed at day 12 after surgery and the implantation is performed at this time, when allodynia is fully established. The delay in allodynia development is associated with inhibition of activation of NF- $\mathrm{\kappa B}$ in

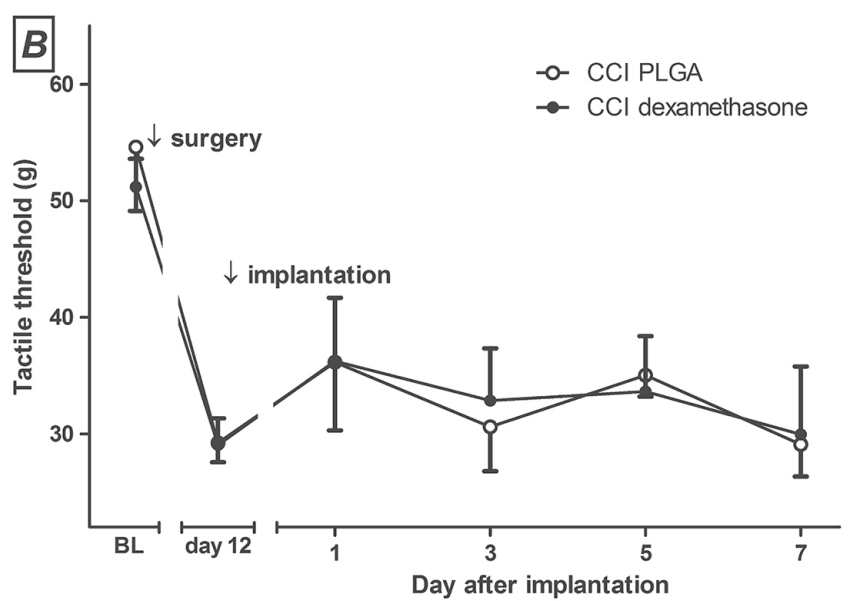

FIGURE 2 - Effect induced by dexamethasone (perineural implant containing $2.4 \mathrm{mg}$, at day 0 (A) or day 12 (B) after constriction of sciatic nerve) on the development of mechanical allodynia in rats. BL: baseline. * statistical difference in comparison with the CCI vehicle (PLGA)-treated group, as revealed by two-way ANOVA followed by Bonferroni post hoc test. (A) Effect of treatment: $\mathrm{F}(1,224)=26.11, \mathrm{p}<0.001$; effect of time: $\mathrm{F}(14,224)=3.81, \mathrm{p}<0.001$; interaction: $\mathrm{F}(14,224)=2.54, \mathrm{p}=0.0022)$. Sham-operated rats were not affected by treatments (all $\mathrm{p}$ values higher than 0.05 ). (B) All $\mathrm{p}$ values higher than 0.05 as well. $\mathrm{n}=6-7$. 

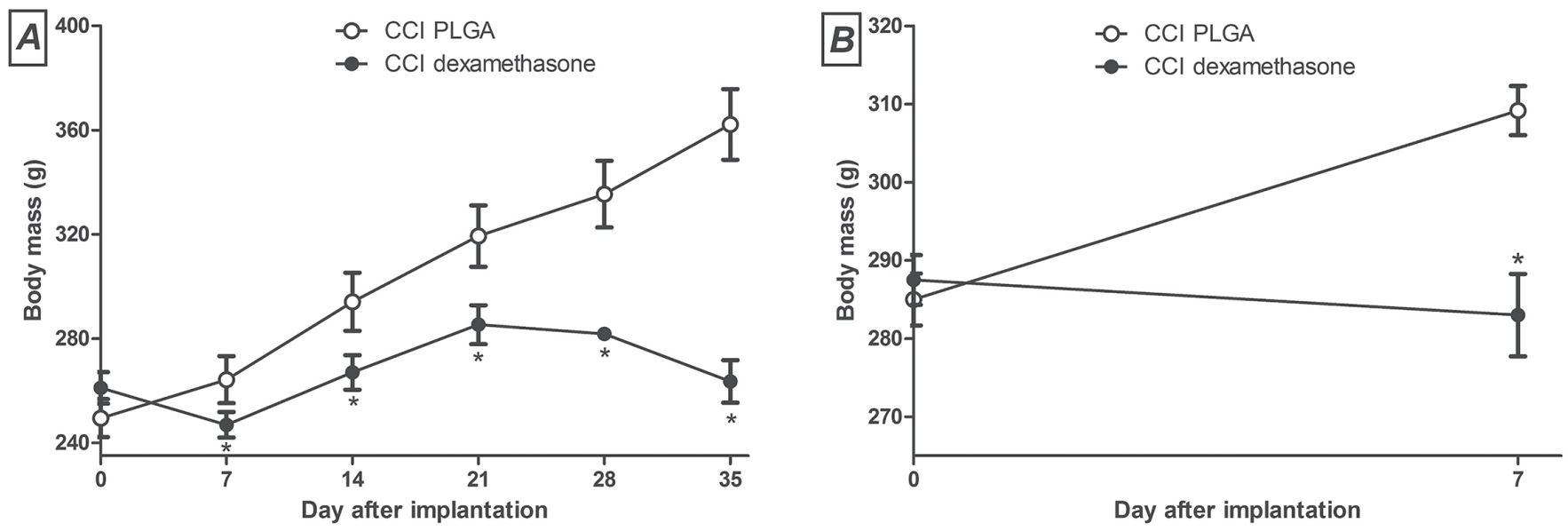

FIGURE 3 - Effect induced by dexamethasone (perineural implant containing $2.4 \mathrm{mg}$, at day 0 (A) or day 12 (B) after constriction of sciatic nerve) on the body mass gain in rats. * statistical difference in comparison with the CCI vehicle (PLGA)-treated group, as revealed by two-way ANOVA followed by Bonferroni post hoc test. (A) Effect of treatment: $F(1,80)=11.25, p<0.004$; effect of time: $F(5,80)=50.96, p=0.0001)$; interaction: $F(5,80)=26.00, p<0.0001$. (B) Effect of treatment: $F(1,10)=5.88, p<0.0357$; effect of time: $\mathrm{F}(1,10)=17.26, \mathrm{p}<0.002$; interaction: $\mathrm{F}(1,10)=36.67, \mathrm{p}=0.0001) . \mathrm{n}=6-9$.
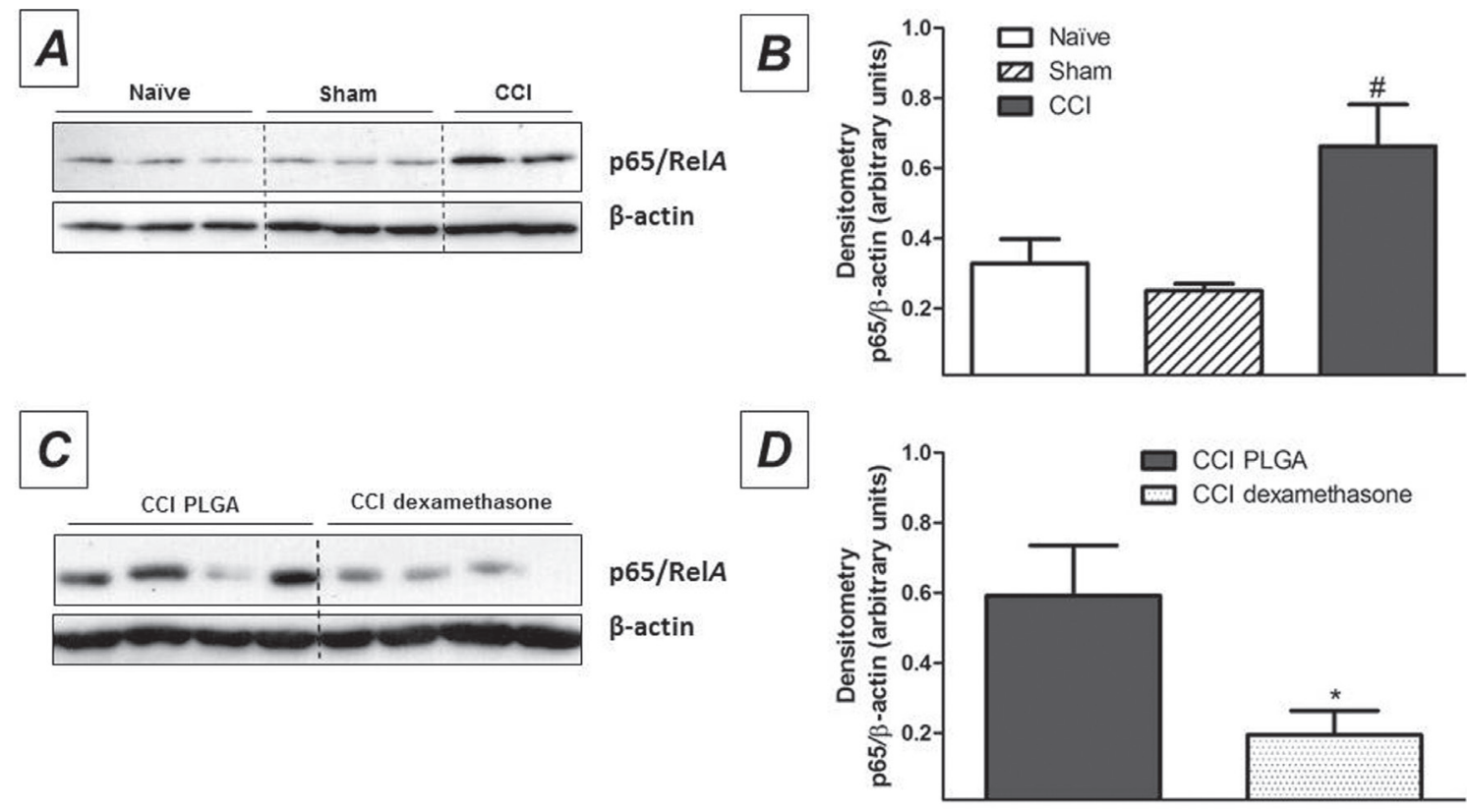

FIGURE 4 - (A and B) Effect induced by chronic constriction injury on the activation of NF-kB in L4-L5 DRG cells (nuclear extract protein content) at day 15 after constriction of sciatic nerve. (C and D) Effect induced by dexamethasone (perineural implant containing $2.4 \mathrm{mg}$, at the moment of constriction, i.e., day 0 ) on the NF- $\mathrm{kB}$ activation. (A and C) Immunoblots. (B and D) Densitometric analysis of corresponding immunoblots. " statistical difference in comparison with the groups naïve and sham (B; $p$ $<0.05$ ), as revealed by ANOVA followed by Newman-Keuls post hoc test. (D) * statistical difference between the two groups (p $<0.05$ ), as revealed by $\mathrm{t}$ student test. $\mathrm{n}=4$.

DRG cells - a peripheral antineuroinflammatory effect. As the dexamethasone implant was placed closely to the CCI site, a local effect is not unlikely and may have contributed to its antiallodynic activity. However, as the perineural dexamethasone implant prevented body mass gain in rats, a systemic effect induced by this drug is evident, thus confirming its well-known catabolic activity
(Bullock et al., 1972) and indicating that effects distant from the site of CCI must also be considered when trying to explain the antiallodynic activity of this steroid.

A previous study showed that the implantation of a depot form of methylprednisolone $(2 \mathrm{mg}$, as acetate) perineurally at day 10 after CCI reverses both mechanical allodynia and thermal hyperalgesia in rats 
(Johansson, Bennett, 1997). The authors of that study hypothesized that the methylprednisolone treatment reduced the hyperexcitability of injured nerve fibers, as a previous study had shown that local methylprednisolone application blocks transmission in normal nociceptive C-fibers (Johansson, Hao, Sjolund, 1990). In vitro studies also suggest dexamethasone's direct actions on neural excitability (Borski, 2000). Local administration of triamcinolone, another SAID, to ferret lingual nerve at the time of injury reduces injury-enhanced, evoked electrical activity in the nerve as long as 3 months after the injury (Yates, Smith, Robinson, 2004). The lack of effect induced by the dexamethasone-loaded implant when mechanical allodynia was fully established is a limitation of the present study. The contrasting effects induced by dexamethasone and methylprednisolone on reversal of ongoing allodynia imply that these SAIDs may act on different biochemical pathways. Further studies are needed to compare their effects on neural excitability in vivo and to evaluate which SAID would be most suitable to be included in an implant aimed to induce a local effect.

The present study indicates that dexamethasone treatment induces peripheral antineuroinflammatory effect, as assessed by inhibition of NF- $\kappa \mathrm{B}$ activation. However, as a systemic effect of dexamethasone is evident, we hypothesize that this steroid may also induce a central pronociceptive effect, as this has been demonstrated elsewhere (Wang et al., 2004), which may counteract, at least partially, the peripheral antineuroinflammatory effect underlying its antinociceptive activity. Dexamethasone may act peripherally through multiple mechanisms to induce the antiallodynic effect. The proinflammatory responses of neutrophils, macrophages, lymphocytes, and mast cells, which normally infiltrate into the nerve after injury (Okuda et al., 2006; Perkins, Tracey, 2000), can be suppressed by dexamethasone (Clatworthy et al., 1995). Furthermore, Schwann cells are activated after CCI, and their production of cytokines and chemokines is inhibited by dexamethasone in vitro (Rutkowski et al., 1999), though in vivo evidence seems to be lacking. This variety of cell types can release a wide array of inflammatory mediators which sensitize or directly activate nociceptors, thereby enhancing pain. Further studies are thus required to elucidate SAIDs' actions on the development and progression of neuropathies.

The results reinforce the evidence that early neuroimmune interactions are essential for triggering mechanisms necessary for the development of long-lasting mechanical allodynia in neuropathic pain models, though additional actions on neural excitability may also be taken into account. Novel therapeutic interventions might be developed based on early suppression of inflammatory response that occurs shortly after a stimulus that causes neuropathic pain.

Although the demonstration of a systemic effect induced by the dexamethasone implant represents another limitation of the present study, the results indicate that the use of this device may allow inducing an effect with lower doses and consequently greater safety. In addition, these results may stimulate further investigation aiming to develop dexamethasone-loaded implants with a better release profile, thus inhibiting only the local changes that contribute to the sensitization induced by CCI and avoiding systemic side effects.

\section{ACKNOWLEDGEMENTS}

The authors wish to thank Fapemig (Brazil), CNPq/ MCT (Brazil), CAPES/MEC (Brazil) and PRPq/UFMG (Brazil) for financial support.

\section{DECLARATION OF INTEREST STATEMENT}

The authors report no declarations of interest.

\section{REFERENCES}

Bennett GJ, Xie YK. A peripheral mononeuropathy in rat that produces disorders of pain sensation like those seen in man. Pain. 1988;33(1):87-107.

Borski RJ. Nongenomic membrane actions of glucocorticoids in vertebrates. Trends Endocrinol Metab. 2000;11(10):427-36.

Bullock GR, Carter EE, Elliott P, Peters RF, Simpson P, White AM. Relative changes in the function of muscle ribosomes and mitochondria during the early phase of steroid-induced catabolism. Biochem J. 1972;127:881-92.

Clatworthy AL, Illich PA, Castro GA, Walters ET. Role of periaxonal inflammation in the development of thermal hyperalgesia and guarding behavior in a rat model of neuropathic pain. Neurosci Lett. 1995;184(1):5-8.

Dash AK, Cudworth 2nd GC. Therapeutic applications of implantable drug delivery systems. J Pharmacol Toxicol Methods. 1998;40(1):1-12.

Fialho SL, Silva-Cunha A. Manufacturing techniques of biodegradable implants intended for intraocular application. Drug Deliv. 2005;12(2):109-16. 
Fu ES, Zhang YP, Sagen J, Candiotti KA, Morton PD, Liebl DJ, et al. Transgenic inhibition of glial NF-kappa B reduces pain behavior and inflammation after peripheral nerve injury. Pain. 2010;148(3):509-18.

Johansson A, Bennett GJ. Effect of local methylprednisolone on pain in a nerve injury model. A pilot study. Reg Anesth. 1997;22(1):59-65.

Johansson A, Hao J, Sjölund B. Local corticosteroid application blocks transmission in normal nociceptive C-fibres. Acta Anaesthesiol Scand. 1990;34(5):335-8.

Kaltschmidt B, Widera D, Kaltschmidt C. Signaling via NF-kappaB in the nervous system. Biochim Biophys Acta. 2005;1745(3):287-99.

Langer R. Drug delivery and targeting. Nature. 1998;392(6679 suppl):5-10.

Ma W, Bisby MA. Increased activation of nuclear factor kappa $\mathrm{B}$ in rat lumbar dorsal root ganglion neurons following partial sciatic nerve injuries. Brain Res. 1998;797(2):243-54.

McMahon SB, Cafferty WB, Marchand F. Immune and glial cell factors as pain mediators and modulators. Exp Neurol. 2005;192(2):444-62.

Motta PG, Perez AC, Alves DP, Duarte ID. Peripheral control of inflammatory but not neuropathic pain by endogenous cholinergic system. Pharmacology. 2011;88(1/2):18-25.
Okuda T, Ishida O, Fujimoto Y, Tanaka N, Inoue A, Nakata $\mathrm{Y}$, et al. The autotomy relief effect of a silicone tube covering the proximal nerve stump. J Orthop Res. 2006;24(7):1427-37.

Perkins NM, Tracey DJ. Hyperalgesia due to nerve injury: role of neutrophils. Neuroscience. 2000;101(3):745-57.

Rutkowski JL, Tuite GF, Lincoln PM, Boyer PJ, Tennekoon GI, Kunkel SL. Signals for proinflammatory cytokine secretion by human Schwann cells. J Neuroimmunol. 1999;101(1): 47-60.

Souza DG, Amaral FA, Fagundes CT, Coelho FM, Arantes RM, Sousa LP, et al. The long pentraxin PTX3 is crucial for tissue inflammation after intestinal ischemia and reperfusion in mice. Am J Pathol. 2009;174(4):1309-18.

Vivancos GG, Verri Jr. WA, Cunha TM, Schivo IR, Parada CA, Cunha FQ, et al. An electronic pressure-meter nociception paw test for rats. Braz J Med Biol Res. 2004;37(3):391-9.

Wang S, Lim G, Zeng Q, Sung B, Ai Y, Guo G, et al. Expression of central glucocorticoid receptors after peripheral nerve injury contributes to neuropathic pain behaviors in rats. J Neurosci. 2004;24(39):8595-605.

Yates JM, Smith KG, Robinson PP. The effect of triamcinolone hexacetonide on the spontaneous and mechanically-induced ectopic discharge following lingual nerve injury in the ferret. Pain. 2004;111(3):261-9.

Received for publication on $28^{\text {th }}$ March 2018 Accepted for publication on $21^{\text {st }}$ June 2018 